\title{
La actitud hacia la docencia, la formación integral en la educación secundaria y a su proyecto de vida: Una visión desde los estudiantes de colegios en áreas rurales
}

\section{Attitude Towards Teaching, Comprehensive Formation, and Students' Life Project: The Point of View of a Group of Students from the $11^{\text {th }}$ Grade in Rural Schools}

\author{
Elionay Quirós Meneses ${ }^{1}$ \\ División de Educología \\ Centro de Investigación y Docencia en Educación \\ Universidad Nacional \\ Heredia, Costa Rica \\ equiroster@gmail.com \\ Xiomara Jiménez Sánchez ${ }^{2}$ \\ Liceo San Jorge de Upala \\ Ministerio de Educación Pública \\ Upala, Costa Rica \\ xiomajs@hotmail.com
}

Recibido 18 de junio de 2012 • Corregido 05 de marzo de 2013 • Aceptado 13 de marzo de 2013

Resumen. Estudio transcenccional descriptivo de enfoque cuantitativo acerca de la actitud hacia la docencia, la formación integral en la educación secundaria y a su proyecto de vida: una visión desde los estudiantes de colegios en áreas rurales, el cual fue realizado en el año 2011. Participaron 41 estudiantes de undécimo año de colegios rurales costarricenses que forman parte del programa Esperanza joven, de la División de Educología de la Universidad Nacional. La información se recopiló con una escala Likert, cuyos resultados permitieron destacar, en los estudiantes, una actitud positiva hacia el desempeño docente, la formación integral, evaluación de los aprendizajes, incentivos familiares e institucionales y metas personales. En tanto, la incentivación que ofrece la institución hacia los estudiantes obtuvo una actitud negativa. Lo anterior permite hacer un análisis objetivo y llegar a conclusiones precisas.

Palabras claves. Actitud, educación, actitud hacia la docencia, incentivación, motivación, Costa Rica, Revista Electrónica Educare.

1 Profesor de la División de Educología del Centro de Investigación y Docencia en Educación de la Universidad Nacional. Labora desde el año 2008 como docente e investigador-extensionista de la División de Educología. Es graduado en Ciencias de la Educación I y II ciclo con Énfasis en Educación Rural. Actualmente labora en el Proyecto Esperanza joven, de la División de Educología.

2 Profesora de enseñanza media (PEM), Bachiller en Estudios Sociales y Cívica, Maestría en Administración Educativa, profesora de los Estudios Sociales en el Colegio Rural de San Jorge de Upala. Ministerio de Educación Pública. 
URL: http://www.una.ac.cr/educare

CORREO: educare@una.cr

\begin{abstract}
This paper refers to a cross-sectional descriptive study with a quantitative approach on the point of view of students from rural areas concerning teaching, comprehensive formation of students from the $7^{\text {th }}$ to $11^{\text {th }}$ grades and the students' life project. The study was conducted in 2011 with the participation of 41 students from the $11^{\text {th }}$ grade of the Costa Rican rural schools involved in the Esperanza Joven program of the School of Educology of the Universidad Nacional. The information was collected through a Likert scale. The results reported a positive attitude towards teacher's performance, comprehensive formation, evaluation of learning, family and institutional stimulus, and personal goals. In contrast, there was a negative attitude about the motivation of the school to its students. This research included an objective analysis and provided accurate conclusions.
\end{abstract}

Keywords. Attitude, education, attitude towards teaching, stimulus, motivation.

El planteamiento de este trabajo tiene como propósito conocer la actitud del estudiantado en relación con la docencia, la formación secundaria y su proyecto de vida en los colegios que participan dentro del programa Esperanza joven.

La perspectiva del estudio se ha enfocado a determinar propiamente la actitud que tienen estos jóvenes dentro del entorno educativo tanto institucional como familiar y el aporte de la familia en el proceso de construcción del proyecto de vida.

Desde hace 12 años, Esperanza joven ha venido realizando una labor trascendental en instituciones educativas, en donde uno de los factores que se ha determinado como influyentes es el aporte de la población educativa, es decir, los diferentes miembros involucrados en el colegio. Y, es precisamente ahí, por datos obtenidos de investigaciones del mismo programa, que se ha detectado la necesidad de una mayor participación de la institución en procesos que motiven y logren despertar, en el estudiantado, un interés por el aprendizaje. Así también, que el apoyo de los miembros de la familia es indispensable para la formación integral de estos jóvenes.

La educación costarricense, a través de los años ha logrado grandes avances en el campo de la educación, por medio de esfuerzos gubernamentales y de otros entes preocupados por una educación de calidad. No obstante, sin quitarles méritos a esos esfuerzos, el sistema educativo costarricense no logra responder a las necesidades y a los intereses de una sociedad tan particular como lo son los estudiantes en la conformación y transformación de su proyecto de vida.

A pesar de los esfuerzos del Estado costarricense a través del Ministerio de Educación Pública y representantes de la educación en general, en muchos lugares del país, especialmente en áreas excluidas como el mundo rural, el impacto de esos esfuerzos se ha desarrollado en forma incompleta. Situación que solo se conoce y valora cuando se está inmerso y se forma parte de esa realidad.

Por ello, es fundamental partir de la realidad educativa y del contexto en la que se encuentran inmersos estos grupos estudiantiles, como entes centrales del proceso educativo.

Para garantizar el conocimiento de esa realidad y su trasformación, al partir de las características y problemáticas que diariamente conviven muchos estudiantes en nuestro 
país, se han desarrollado diferentes tipos de actividades que involucran a las comunidades educativas en la búsqueda de soluciones para transformar su realidad. Por eso, uno de los aportes más importantes del programa Esperanza joven es desarrollar una actitud crítica y creativa del estudiantado ante el proceso educativo, de tal manera que logre replantearse su proyecto de vida desde la perspectiva de la formación brindada por la educación secundaria.

Esto lleva a realizar un análisis de sus actitudes, la docencia, la formación integral y su proyecto de vida en el año 2011, con el propósito de valorar el aporte educativo que reciben, desde la perspectiva de los jóvenes y las jóvenes que cursan quinto año de la secundaria en los colegios de Villanueva, Las Delicias y San Jorge de Upala, ubicados en la zona norte del país, cerca de la frontera con Nicaragua.

Las razones antes expuestas determinan el propósito de este estudio, el cual se caracteriza por valorar las actitudes hacia la docencia, formación integral y el proyecto de vida.

Una manera de conocer la realidad es partir de los actores considerados como centro del proceso educativo, es decir, los estudiantes y las estudiantes, pues eso va a permitir valorar su criterio sobre la educación recibida en instituciones de educación secundaria.

\section{Referente teórico}

La educación como fenómeno social ha contribuido en la transformación de la humanidad a tal punto que hoy día se pueden evidenciar cómo el ser humano ha logrado cambios significativos en el desarrollo y la calidad de vida.

Sin duda, es fundamental que se continúe trabajando arduamente en la búsqueda constante de nuevas formas de transformar y de impactar a la mayoría de los seres humanos, de tal forma que un mundo desequilibrado -donde no todas las personas tienen acceso a la educación con, al menos, las condiciones básicas, a pesar de enfrentarnos a un siglo XXI con grandes oportunidades, con accesos inmediatos a las nuevas tecnologías de la información y la comunicación- se pueda convertir y revertir a derechos propiamente humanos para romper con esa desigualdad social.

Desde ese punto de vista, junto a otros factores que también inciden y marcan el interés en el ser humano, ya sea porque le favorecen o repercuten negativamente en él, se pretende analizar y buscar alternativas inteligentes que den garantía de una educación con sentido para todos y todas.

La realidad antes mencionada condiciona para que muchos jóvenes terminen desertando del sistema educativo formal, y le den paso a otras actividades, en su proyecto de vida, las cuales no corresponden a las que, conscientemente, desearían para sí.

La motivación y el interés de una persona radica, primordialmente, en las oportunidades a las que pueda acceder en su transformación y reconocimiento desde el punto de vista que 
URL: http://www.una.ac.cr/educare

CORREO: educare@una.cr

tenga de sí mismo. En ciertos casos, esa motivación se logra de una manera natural, denominada como motivación intrínseca, o por medio de la dependencia de otras situaciones e individuos ajenos a la persona en sí, tal como el apoyo que reciban de la institución educativa, los padres y madres de familia, el profesor o la profesora, las relaciones interpersonales entre compañeros y compañeras y, además, con gran relevancia la autoconciencia por aprender para la vida.

Alonso y Montero (1990) enfatizan en que "(...) un estudiante se encuentra motivado o desmotivado cuando se enfrenta a numerosas situaciones que tienen que ver con el contexto familiar, educativo, social, emocional y/o psicológico, entre otras (p. 185)".

Dada la importancia de la motivación -tanto intrínseca como extrínseca- para que los jóvenes permanezcan dentro del sistema educativo, con metas de culminar con éxito la educación secundaria, existen elementos internos y externos dentro de cada institución que condicionan y determinan la actitud estudiantil hacia la educación recibida.

Considerando lo anterior, algunos aspectos fundamentales necesarios de tomar en cuenta en el desarrollo de esas actitudes de estudiantes de educación secundaria en un contexto rural son el desempeño docente, la formación integral del estudiantado, la evaluación de los aprendizajes, la incentivación desde la institución educativa, los incentivos familiares y metas personales de los estudiantes y las estudiantes. De allí la necesidad de conceptualizar esos aspectos.

Considerando que este consiste en un estudio actitudinal, es de gran relevancia conceptualizar el término actitud para orientar y sustentar el aporte desde los criterios seleccionados en este estudio.

Una persona con un estado motivacional elevado, por lo general enfrenta retos que son claves en la vida. Muy de la mano a lo anterior, se puede decir que la actitud es un componente que funciona como agente transformador en el ser humano y que marca las pautas en el estado anímico y su forma de ver la realidad del mundo. El estado actitudinal se puede concebir como aquel estado emocional que, según sea la situación, puede variar de acuerdo con el nivel de madurez, a la par del conjunto de experiencias de aprendizaje que logre una persona. O bien, es lo que se puede descifrar como la manera en la cual el ser humano se puede comunicar desde el estado de ánimo en que se encuentre en relación con las demás personas (Hill y Stone 2003).

Hay ciertas actitudes que se consideran relevantes para enfrentar el estudio con una actitud positiva. Una de ellas es que el estudiante defina con claridad cuáles son las metas que se propone a corto, mediano y largo plazo; asimismo, los objetivos que quiere lograr; reconocer que el docente o la docente es una persona profesional que desempeña una tarea especial en su proceso de formación y que ello significa darle un valor especial, prestarle atención a lo que considera beneficioso para sus estudios; confiar en sí mismo, y siempre tener presente que día tras día se puede mejorar en todas las dimensiones, por lo tanto, estar en constante autoanálisis para valorar lo que necesita cambiar para bien. 


\section{El desempeño docente}

El desempeño docente se puede considerar como las sumas de acciones que desarrolla un docente para el logro de los objetivos educativos de manera integral y fundamentada en principios de una profesión comprometida con el desarrollo humano y el desarrollo del conocimiento científico, razón por la cual:

El desempeño docente laboral califica la calidad profesional con la que se espera que haga el profesor de educación, mide variadas cualidades como pueden ser: Hombre mujer, conductas en el trabajo, puntualidad, comportamiento, disposición para el trabajo, disciplina, compromiso institucional, desarrollo pedagógico, innovación. (De la cruz, 2007, p. 5)

Junto a esas acciones que le corresponden al docente, la tarea principal, sin duda alguna, es favorecer el desarrollo y el progreso de sus estudiantes en su formación integral. Tal como lo afirma Muñoz (2010): "(...) Su función es mediar y asistir en el proceso por el cual lo estudiantes desarrollan sus conocimientos, sus capacidades, sus destrezas, actitudes y valores, en el marco de un comportamiento que valora a otros y respeta los derechos individuales y sociales" (párr. 5).

Es evidente que el personal docente, en su práctica profesional, debe tomar en cuenta su actuación y la comprensión de la responsabilidad que le demanda, pues es una labor indispensable que permite el progreso y el desarrollo en las personas y, por consiguiente, en la sociedad.

Se pueden reconocer algunas características que demarcan el perfil en el desempeño docente. Estas son la producción docente, referida al aporte científico y al desarrollo de la producción intelectual desde la investigación y las prácticas vividas dentro y fuera del aula escolar; el perfeccionamiento docente, orientado a la capacitación, actualización y al desenvolvimiento que se desprenda para mejorar su desempeño profesional y, finalmente, la proyección a la comunidad, pensada desde la perspectiva de los aportes que puedan dar estos profesionales para contribuir en la solución de los problemas y para satisfacer las demandas de las comunidades (Carranza, 2005).

Desde este punto de vista, Reyes, L. (2006) insiste en la importancia de:

(...) precisar que el profesor o profesora ya no es únicamente el servidor de un EstadoNación que educa a unos y deja fuera a otros. Su trabajo se dirige a una sociedad crecientemente diversa y plural, que demanda para todos sus jóvenes un tipo de educación que los prepare, no sólo para su participación ciudadana, sino también para su participación productiva (...). (p. 2)

Y es allí donde se marca el desafío del profesor o profesora de enfrentar nuevas demandas que garanticen un mejor desempeño docente acorde con las necesidades y retos del siglo XXI. 
URL: http://www.una.ac.cr/educare

CORREO: educare@una.cr

\section{La formación integral del estudiante}

Desde la complejidad de la educación como fenómeno transformador en el ser humano, se puede decir que el ser humano aprende desde diversas formas: desde la formación que reciben en el hogar; desde las experiencias cotidianas; desde la formación académica, vista como la responsabilidad que le compete a la escuela como institución formadora; desde la formación religiosa; entre otras, que siguen siendo de gran relevancia.

Esos aprendizajes van definiendo a la persona como lo que quiere ser desde los principios que le identifican como persona. De allí, todo lo que se aprende en la vida y para esta es lo que resulta relevante para su desarrollo y la formación.

De manera concreta, desde la perspectiva social, "la [negritas añadidas] Formación Integral es entendida como un proceso continuo, permanente y participativo que busca desarrollar armónica y coherentemente todas y cada una de las Dimensiones del [negritas añadidas] ser humano a fin de lograr su realización plena en la sociedad (Gaitán et al., p. 64).

Por tanto, con base en estos aportes, el ser humano aprende para la vida, aprende a ser ciudadano y ciudadana responsables con sus actos; aprende a hacer y proponer, aprende a contribuir con la sociedad y, por ende, con el progreso y el bienestar de la gente.

Precisamente, desde esos criterios se percibe que la educación es integral y que busca la formación de los individuos para un mejor desenvolvimiento en la sociedad, para lograr mejor calidad de vida.

Espinal, A. (2003) propone un esquema que reúne algunos elementos esenciales en la formación integral de la persona. Estos son:

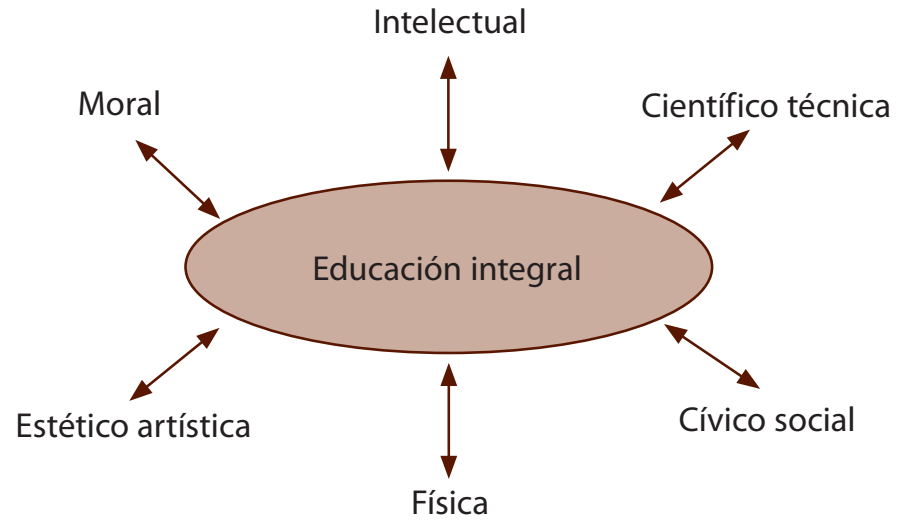

Figura 1. Elementos de la educación integral. Tomado de "La educación integral en las escuelas secundarias" de Espinal (2003), p. 2. 
Con este mismo fin, es preciso citar que la educación costarricense se ampara en los siguientes fines que reflejan los principios elementales garantes de la formación integral del estudiantado, tal como lo menciona el Ministerio de Educación Pública, República de Costa Rica (2007).

Artículo 2.- Son fines de la educación costarricense:

a) La formación de ciudadanos amantes de la patria, conscientes de sus deberes, de sus derechos y de sus libertades fundamentales, con profundo sentido de responsabilidad y de respeto a la dignidad humana.

b) Contribuir al desenvolvimiento pleno de la personalidad humana.

c) Formar ciudadanos para una democracia en que se concilien los intereses del individuo con los de la comunidad.

d) Estimular el desarrollo de la solidaridad y de la comprensión humanas.

e) Conservar y ampliar la herencia cultural, impartiendo conocimientos sobre la historia del hombre, las grandes obras de la literatura y los conceptos filosóficos fundamentales.

Artículo 3.- Para el cumplimiento de los fines expresados, la escuela costarricense procurará:

a) El mejoramiento de la salud mental, moral y física del hombre y de la colectividad.

b) El desarrollo intelectual del hombre y sus valores, estéticos y religiosos.

c) La afirmación de una vida familiar digna, según las tradiciones cristianas, y de los valores cívicos de la democracia.

d) La transmisión de los conocimientos y técnicas, de acuerdo con el desarrollo psicológico de los educadores.

e) Desarrollar aptitudes, atendiendo adecuadamente las diferencias individuales.

f) El desenvolvimiento de la capacidad productora y de la eficiencia social. (pp. 1-2)

Es decir, como país, en Costa Rica este tema se considera de gran relevancia, dado que, en la actualidad, la formación de la persona debe orientarse tomando en cuenta todo su potencial, sus habilidades, sus conocimientos, para el disfrute de una mejor calidad de vida. Desde esa perspectiva, el estudiantado debe incorporar algunos componentes que en su conjunto forman parte de un sistema esencial desde la formación integral. Entre ellos: hábitos positivos tanto personales como académicos; actitud propositiva ante la vida; principios y valores positivos que le identifiquen como individuo social, lleno de cualidades, capaz de socializar y de involucrarse en la sociedad. 
URL: http://www.una.ac.cr/educare

CORREO: educare@una.cr

\section{La evaluación de los aprendizajes}

La evaluación, la enseñanza y el aprendizaje deben constituirse en una unidad indisoluble (Coll, Martin y Onrubia, 2001), de manera tal que la evaluación sirva para explicar tanto los aprendizajes de los estudiantes y las estudiantes como la actividad instruccional de su docente.

Por tanto, estos elementos son esenciales y permiten, de una manera objetiva, sistematizada y con plena organización, comprender que la educación es un proceso y, por ende, las funciones que cumple cada una de estas unidades resulta de suma importancia.

En relación con la tarea de la evaluación en los procesos de aprendizaje, esta debe asumirse desde una perspectiva holística:

La finalidad de la evaluación de los aprendizajes es valorar y analizar, tomando decisiones las cuales impactan directamente a los educandos comprometiendo de forma ética al evaluador en este caso al educador ya que de su formación y de su profesionalismo dependen la obtención de aprendizajes significativos en cada uno de los niños y niñas. (García, K., 2010, p. 25)

Por tal razón, y más aún en Costa Rica, este tema debe ser cuidadosamente valorado, dado el impacto y lo que significa dentro del proceso de aprendizaje. Si no hay objetividad $y$, por ende, conciencia en la forma en que se evalúan los aprendizajes, al final puede ser que como consecuencia hallan individuos desertores del sistema educativo, desmotivados $y$, por consiguiente, sin ningún interés para continuar sus estudios.

\section{La incentivación desde la institución educativa}

Hay que partir de que no todo estudiante logra motivarse en los estudios y, desde esa perspectiva, la institución educativa tiene un papel fundamental para lograr su entusiasmo por aprender, de tal manera que el interés de este individuo, cada vez sea mayor en su formación integral. Por lo general, hay ciertas instituciones educativas que buscan alternativas para incentivar a sus estudiantes en la educación para que alcancen estar motivados.

Por medio de la Resolución № 0229 de Dic. 03/99 de la Secretaría Distrital de Educación y Cultura de Cartagena, se ha tomado como política institucional incentivar a los estudiantes las estudiantes a través de estímulos que ayuden a mejorar sus actitudes y que ello les implique hacer la diferencia en su rendimiento académico, cultural, deportivo o científico y a nivel personal. 
Estos incentivos son los siguientes:

- Premiación, por caritas felices, banderas, flores, dulces, sellos.

- Ubicarlos en un Tabla de honor.

- Entregar menciones de honor y medallas, al final del año académico.

- Promoción a un grupo superior dentro del mismo periodo lectivo.

- Entregar becas al final del año lectivo, para el año siguiente. (Resolución № 0229 (1999, párr. 2-6)

Estos estímulos vienen a ser puntuales cuando uno, o bien un grupo de estudiantes, logran ciertos honores y la institución educativa favorece a dichos estudiantes dándoles méritos y reconociéndoles virtudes. Esto favorece que le encuentren sentido para la vida, a la formación integral que reciben.

Con base en una visión más integral, a nivel de políticas institucionales, la Institución Educativa Técnica Comercial de Caldas, por medio del Proyecto educativo institucional, considera que, desde el papel que desempeña una institución educativa en la incentivación de los procesos de aprendizaje, hay que partir de las siguientes estrategias:

- Implementar metodologías activas, creativas e innovadoras, y así fortalecer el proceso metodológico, y mejoramiento de las destrezas de los estudiantes.

- Mejorar la metodología de trabajo para hacer que los estudiantes se interesen mas por aprender que por la calificación.

- Diseñar actividades significativas y valiosas de parte del Docente.

- Seguimiento a estudiantes con aprendizaje lento.

- Conocer el estado de motivación intrínseca y Extrínseca [sic] que maneja el del estudiante.

(...)

- Proporcionar un clima institucional agradable-sin presión. (párr. 4-10)

\section{Propuesta metodológica}

Este es un estudio realizado dentro del paradigma positivista, con un enfoque cuantitativo y un diseño transcenccional descriptivo, realizado en tres colegios rurales de la zona norte de Costa Rica: Villanueva, Las Delicias y San Jorge de Upala. 
URL: http://www.una.ac.cr/educare

CORREO: educare@una.cr

La población estudiada son 41 estudiantes de ambos sexos que cursaban quinto año en el año 2011 y cuyas edades oscilan entres 17 y 22 años.

Por lo cercano con la frontera de Nicaragua, algunos estudiantes son migrantes de dicho país.

Acorde con el colegio de procedencia, la población participante en este estudio se distribuyó de la siguiente manera:

Tabla 1

Instituciones participantes en el estudio y su distribución según

el colegio

\begin{tabular}{lc}
\hline Nombre del colegio & Cantidad de estudiantes \\
\hline Villanueva & 9 \\
Las Delicias & 18 \\
San Jorge & 14 \\
\hline Total & 41 \\
\hline
\end{tabular}

Nota. Tomado de los instrumentos facilitados a los participantes.

Como se puede observar en este estudio, por el tamaño de la población no se utiliza muestra.

Las variables tomadas en cuenta para el estudio fueron las siguientes:

El desempeño docente, la formación integral del estudiante, la evaluación de los aprendizajes, la incentivación desde la institución educativa y los incentivos familiares y metas personales del estudiantado, las cuales fueron medidas mediante una escala de actitud que tenía como máximo cuarenta preguntas y cuyos resultados fueron interpretados tomando como referente la tabla 2:

Tabla 2

Escala para integrar los resultados del instrumento

\begin{tabular}{|llll|ll|}
\hline Actitud negativa & & Sin criterio & & Actitud positiva & \\
\hline 1 & 2,59 & 3 & 3,59 & 3,60 & 5
\end{tabular}


Lo anterior se deriva de que las opciones en cada pregunta del instrumento eran las siguientes: "Totalmente de acuerdo", con un valor de 5 puntos; "De acuerdo", equivalente a 4 puntos; "Sin criterio", igual a 3 puntos; "En desacuerdo", con valor de 2 puntos, y "Totalmente en desacuerdo", con 1 punto.

Los pasos dados para la realización del estudio se llevaron a cabo dentro de las primeras visitas hechas a los colegios respectivos dentro del plan de trabajo del programa Esperanza joven, en el año 2011, donde se aplicó la escala de actitud.

Una vez recabada la información, se tabularon los datos, se hicieron los cálculos respectivos sobre la actitud hacia cada una de las variables mencionadas $y$, con base en esa información, se sistematizaron los datos estadísticos y, desde allí, se interpretaron los resultados.

\section{Análisis discusión e interpretación de los resultados}

Los principales resultados obtenidos en este estudio son los siguientes:

Tabla 3

Actitudes favorables, desfavorables o neutras ante el desempeño docente del estudiantado de tres liceos rurales en el año 2011

$(N=41)$

1. La mayoría de docentes mantiene comunicación asertiva con el estudiantado.

2. La mayoría de docentes muestra preocupación para que el estudiantado aprenda.

3. La mayoría de docentes trata mal al estudiantado.

4. El profesorado guía mantiene comunicación con los padres o madres de familia.

5. Las actividades realizadas en clase contribuyen a aprender la materia.

6. Las clases se imparten en forma expositiva/magistral.

7. Los resultados de la evaluación realizadas por el personal docente comunican realmente lo aprendido.

8. En las clases, como estudiantes, podemos participar activamente.

9. Nuestros profesores y profesoras nos incentivan a que triunfemos como estudiantes.

Nota: Escala de actitudes hacia la docencia, la formación en educación secundaria y a su proyecto de vida. 
URL: http://www.una.ac.cr/educare

CORREO: educare@una.cr

De la información obtenida en la tabla 3, el desempeño docente es positivo. Sin embargo, cabe destacar que con respecto al punto seis, referido al desarrollo de las clases, lo que priva es la clase magistral o expositiva. Por ello, obtiene una actitud negativa hacia la misma.

Cabe mencionar que en relación con los puntos uno, dos y cuatro, el estudiantado mantiene una actitud neutra. Esto podría ser porque sus profesores o profesoras no han logrado la empatía necesaria que requiere la relación profesor-estudiante y que garantice la relación asertiva entre los actores directos e indirectos del proceso educativo.

También es relevante destacar que el estudiantado muestra una actitud positiva hacia la participación que permite el personal docente dentro del aula (ver punto ocho) que es la actitud más positiva dentro de esta tabla 3.

A pesar de que tienen una actitud negativa ante las clases magistrales, si mantienen una actitud sobresaliente hacia la participación. Es posible, entonces, la combinación entre clases magistrales y lecciones donde los estudiantes tengan la oportunidad de participar activamente.

Tabla 4

Actitudes favorables, desfavorables o neutras ante la formación integral del estudiantado de tres liceos rurales en el año 2011

$(N=41)$

\section{Aspectos}

1. En el colegio nos preparan para tener éxito en la vida.

2. La orientación vocacional brindada el colegio nos permite tener claro qué debemos hacer al terminar el quinto año.

3. La mayoría de los(as) profesores (as) nos enseñan a aprender a aprender.

4. Las experiencias de aprendizaje desarrolladas en el colegio me enseñan a pensar.

5. En el colegio he aprendido a enfrentar y decir no a las drogas.

6. En el colegio he aprendido a enfrentar y decir no al alcohol.

7. En el colegio he logrado aprender conocimientos fundamentales sobre educación sexual.

Promedio general

Nota: Escala de actitudes hacia la docencia, la formación en educación secundaria y a su proyecto de vida.

Dentro de lo que se puede observar en la tabla 4, se denota que hay una tendencia general en el grupo hacia la actitud positiva sobre la formación integral recibida, ya que la mayoría de las actitudes relacionadas con la categoría en estudio se manifiestan positivas. Al tomar como referente la tabla para interpretar los resultados de la escala de actitudes, la tabla 4 muestra que no existen rubros que determinen que el grupo no tenga criterio para valorar la actitud (neutra). 
No obstante, sí es indispensable prestar atención con respecto a la actitud hacia los procesos de orientación vocacional, uno de los rubros (el dos) que aparece más bajo. Esto parece indicar que este aspecto sigue siendo una necesidad de atención en las instituciones educativas.

Tabla 5

Actitudes favorables, desfavorables o neutras ante la evaluación de los aprendizajes del estudiantado de tres liceos rurales en el año 2011

$(N=41)$

1. La evaluación que desarrollan la mayoría de docentes es justa.

2. La evaluación de los diferentes cursos se fundamenta solo en el dominio de contenidos de las materias.

3. La evaluación aplicada por el personal docente solo mide el aprendizaje memorístico.

4. Los resultados de la evaluación solo me interesan para ganar el curso.

5. Los resultados de la evaluación me interesan porque conozco lo que realmente he aprendido.

6. Los resultados de la evaluación los comunico a mi padre, madre o encargado.

7. Mis padres se preocupan por saber los resultados de mis evaluaciones de las diferentes materias.

8. Los resultados de la evaluación de mis materias los uso para determinar qué debo mejorar y así estudiar más.

Nota: Escala de actitudes hacia la docencia, la formación en educación secundaria y a su proyecto de vida.

Con respecto a la actitud que tienen los estudiantes hacia la evaluación de los procesos de aprendizaje dentro de las instituciones, es importante resaltar que de ocho actitudes determinadas, solo tres de ellas alcanzan una calificación positiva hacia dichos procesos. Esto demuestra que, a pesar de que el promedio de las actitudes manifestadas determina que hay una actitud positiva, el resultado de ese promedio está muy cercano a la ausencia de criterio para responder $(3,67)$, dado que para cinco de las actitudes medidas, el estudiantado manifiesta no tener el criterio para hacerlo (neutro). Además, las actitudes positivas tienen un puntaje alto, sobre todo en la labor que realizan los padres y madres de familia y el interés de los estudiantes y las estudiantes por aprender.

Lo anterior demuestra que no existe en las instituciones una cultura que valore el proceso y los resultados de la evaluación tal como lo estipula lo normado por el Ministerio de Educación Pública. Por lo tanto, exige una clarificación del aporte de la evaluación y sus resultados para realimentar el logro de los objetivos de aprendizaje. 
URL: http://www.una.ac.cr/educare

CORREO: educare@una.cr

Tabla 6

Actitudes favorables, desfavorables o neutras ante la incentivación desde las instituciones educativas al estudiantado de tres liceos rurales en el año 2011

$(N=41)$

1. El plan de becas del colegio ayuda a quienes más lo necesitan.

2. En el colegio nos orientan para que logremos solicitar una beca.

3. El servicio de biblioteca nos apoya para que tengamos éxito en el estudio.

4. Las competencias deportivas dentro del colegio me motivan a ser parte de la institución.

5. El servicio de comedor es de calidad en mi colegio.

6. El colegio realiza intercambios estudiantiles con otras instituciones.

7. El servicio de orientación del colegio contribuye para tener claro mi proyecto de vida luego que termine la educación secundaria.

8. Nadie me ayuda a orientarme para tener éxito en mi vida al terminar el colegio.

Nota: Escala de actitudes hacia la docencia, la formación en educación secundaria y a su proyecto de vida.

En la tabla 6 anterior se muestra la actitud de los estudiantes hacia la incentivación que reciben desde las instituciones educativas. Se observa que es una de las categorías que presenta un promedio general de 2,8. Es decir, acorde con la tabla de interpretación, los estudiantes en promedio tienen una actitud negativa hacia esos incentivos.

Por lo anterior, es importante destacar que en cada una de las manifestaciones actitudinales que componen la categoría, la mayoría muestra puntajes muy bajos, es decir, que se puede interpretar como negativos. Entre ellos, el apoyo que reciben del servicio de biblioteca, el desarrollo de la vida estudiantil en la institución, (competencias e intercambios) y hacia los servicios como el de comedor y el de orientación. Lo único que se resalta como un aspecto relevante en esta categoría es la orientación que reciben los estudiantes para solicitar la beca estudiantil.

Las actitudes manifestadas por el estudiantado resaltan las debilidades de las instituciones educativas estudiadas para lograr incentivar a sus estudiantes a mantenerse dentro del sistema educativo. Estos resultados exigen a estas instituciones cambios en sus políticas para alcanzar un mejoramiento hacia esas actitudes positivas, de tal manera que sus estudiantes se sientan incentivados para continuar sus estudios. 
Tabla 7

Actitudes favorables, desfavorables o neutras ante los incentivos familiares y metas personales del estudiantado de tres liceos rurales en el año 2011

$(N=41)$

1. Mi familia me brinda apoyo económico para mis estudios.

2. El afecto que me brinda mi familia contribuye a ser mejor estudiante.

3. En casa me apoyan para que realice mis tareas escolares.

4. Mi familia me apoya para que siga estudiando al terminar el quinto año.

5. Lucho por el éxito en mis estudios por mis planes que tengo para mi vida.

6. Participo en actividades de la institución que contribuyan a mejorar la calidad de mi educación.

7. Me preocupo por lograr buenos resultados en mis estudios.

8. Tengo claro lo que deseo ser en mi vida.

Nota: escala de actitudes hacia la docencia, la formación en educación secundaria y a su proyecto de vida.

Con respecto a la actitud que asumen los estudiantes ante los incentivos familiares y metas personales, el promedio general muestra una actitud positiva. Es decir, tanto la familia como el estudiantado están actitudinalmente positivos para el reto que les exige el estudio.

Cabe destacar que las dos actitudes negativas en esta categoría son: lo que deseo ser en mi vida y la participación en las actividades de la institución para mejorar la calidad de la educación. Esta situación se suscita, posiblemente, como las personas informantes lo plantearon, por las deficiencias del servicio de orientación y la poca incentivación que las instituciones brindan al estudiantado para que mejoren la calidad de su educación.

Sí resulta importante considerar la actitud positiva hacia el aporte de la familia para que los estudiantes y las estudiantes tengan éxito dentro del proceso educativo que ofrece la institución. Es decir, las familias cifran sus esperanzas y brindan el apoyo para el éxito de sus hijos e hijas con la educación. 
URL: http://www.una.ac.cr/educare

CORREO: educare@una.cr

Tabla 8

Promedio general de las categorías y su relación con las actitudes del estudiantado ante la educación

\begin{tabular}{lcc}
\hline Actitudes por categoría & Promedio & Tipo de actitud \\
\hline $\begin{array}{l}\text { Actitudes favorables, desfavorables o neutras ante el desempeño docente, } \\
\text { según el estudiantado de tres liceos rurales en el año 2011. }\end{array}$ & 3,64 & Positiva \\
$\begin{array}{l}\text { Actitudes favorables, desfavorables o neutras ante la formación integral del } \\
\text { estudiantado de tres liceos rurales en el año } 2011 .\end{array}$ & 3,98 & Positiva \\
$\begin{array}{l}\text { Actitudes favorables, desfavorables o neutras ante la evaluación de los } \\
\text { aprendizajes del estudiantado de tres liceos rurales en el año 2011. }\end{array}$ & 3,67 & Positiva \\
$\begin{array}{l}\text { Actitudes favorables, desfavorables o neutras ante la incentivación desde las } \\
\text { instituciones educativas al estudiantado de tres liceos rurales en el año 2011. }\end{array}$ & 2,8 & Negativa \\
$\begin{array}{l}\text { Actitudes favorables, desfavorables o neutras ante los incentivos familiares y } \\
\text { metas personales del estudiantado de tres liceos rurales en el año } 2011 .\end{array}$ & 3,98 & Positiva \\
\hline \begin{tabular}{l} 
Promedio total de la escala \\
\hline
\end{tabular} & 3,65 \\
\hline
\end{tabular}

Nota: Escala de actitudes hacia la docencia, la formación en educación secundaria y a su proyecto de vida.

La tabla 8 permite observar los resultados obtenidos sobre el promedio actitudinal grupal entre las cinco categorías que conforman la escala de actitud. (Promedio general, 3,65) Los resultados de la escala determinan que, en términos generales, el estudiantado muestra una actitud positiva ante el desempeño docente, la formación recibida en la institución y el proyecto de vida forjado desde esta.

Sin embargo, se resalta que la única actitud negativa manifestada es la incentivación que reciben de la institución educativa para continuar exitosamente el proceso de aprendizaje. Esta calificación marca un reto para las instituciones participantes en este estudio. Es un rubro que deben incorporar prioritariamente dentro del Proyecto Educativo Institucional, como medida para lograr mayor incentivación en la población estudiantil, para que rinda académicamente y en su desarrollo personal logren desempeñarse mejor. Así se apostaría por logros en su condición tanto como estudiantes y como de seres humanos, lo que atrae, como consecuencia, motivación intrínseca para estudiar y, por ende, para ir logrando metas dentro de su proyecto de vida.

Otra información digna de tomar en cuenta como relevante es la actitud positiva que manifiestan los estudiantes hacia la formación integral que reciben en la institución, y los incentivos familiares y metas personales. Si se visualizan las tablas 4 y 7 se evidencia lo positivo de las actitudes de los estudiantes hacia el aporte de familia.

A pesar de puntuar alto el promedio actitudinal hacia la formación integral, estas se ven afectadas con la actitud que asumen los estudiantes ante la evaluación, pues a pesar de 
considerarse positiva, el puntaje está cercano a no tener criterio para valorar esta actitud, situación que se repite con el desempeño docente.

Por lo anterior, podría considerarse que a pesar de la actitud positiva de los estudiantes hacia la evaluación y el desempeño docente, estos son dos aspectos que necesariamente tienen que ser reforzados para que la actitud de los estudiantes sea más clara, pero sobre todo positiva. Este reto será asumido desde la capacitación al personal docente, para que logre ser lo más asertivo el proceso de evaluación de los aprendizajes y en los procesos de enseñanza y aprendizaje.

\section{Conclusiones}

Con base en el análisis de los resultados se establecen las siguientes conclusiones:

\section{En general}

En términos generales, el estudiantado manifiesta una actitud positiva hacia la docencia, la formación integral y al proyecto de vida.

\section{El desempeño docente}

La actitud estudiantil hacia el desempeño es positiva. Sin embargo, hay aspectos particulares de este rubro que deben ser mejorados, dado que a pesar de mostrarse como positivos no alcanzaron porcentajes altos.

Tal es el caso del desarrollo de las lecciones que se imparten de manera magistral. No obstante, a pesar de la actitud negativa ante las clases magistrales, el estudiantado mantiene una actitud sobresaliente hacia la oportunidad que dan los docentes de participación en clase.

\section{La formación integral del estudiantado}

La actitud que prevalece en el estudiantado es positiva hacia la formación integral que reciben en la institución. Empero, hay que ponerle atención al mejoramiento de los procesos de orientación vocacional dentro de las instituciones involucradas en el estudio.

\section{La evaluación de los aprendizajes}

No existe claridad en cuanto a la importancia y el aporte que tiene la evaluación para realimentar los procesos de enseñanza y aprendizaje.

Se requiere tener más entendimiento del qué y el para qué del proceso de evaluación, tanto en el desarrollo de las clases como en el producto que se alcanza dentro de la institución, para así lograr realimentar los objetivos educativos y de aprendizaje. 
URL: http://www.una.ac.cr/educare

CORREO: educare@una.cr

Cabe destacar que los padres y madres de familia son los más interesados en los resultados del proceso evaluativo de los aprendizajes de sus hijos e hijas en la institución, a pesar del poco aporte brindado por la cultura de la evaluación en la institución, pues no se tiene claro para qué se evalúa, de allí la indecisión del estudiantado alrededor de este tema.

\section{La incentivación desde la institución educativa}

La incentivación que ofrece la institución educativa hacia el estudio es considerada como una de las variables que reflejan una actitud negativa, lo cual determina la existencia de una problemática institucional que no responde a las necesidades sus estudiantes para el logro de mayor motivación en sus estudios.

Esta carencia en el tema de incentivación, destacada por la actitud asumida por el estudiantado, refleja que la institución educativa presenta planes que no le incentivan a encontrar, en la institución, un espacio para crecer personal y académicamente: quizá las instituciones tienden a limitarse a la acción de dar clases sin pensar que el proceso educativo debe ser integral.

\section{Los incentivos familiares y metas personales de los estudiantes}

La actitud del estudiantado hacia la incentivación que reciben de la familia y sus metas personales es positiva. Por ello, se destaca la familia como un apoyo importante para desarrollar esa actitud, más que lo aportado por la institución. Eso viene a reforzar las razones por las cuales los estudiantes y las estudiantes puntúan bajo el aporte de la orientación y los incentivos institucionales.

\section{Referencias}

Alonso, J. y Montero, I. (1990). Motivación y aprendizaje escolar. En C. Coll, J. Palacios y Á. Marchesi (Comps). Desarrollo psicológico y educación 2: Psicología de la educación escolar (2a ed., pp. 183-198). Madrid: Alianza Editorial.

Carranza, G. (2005). El desempeño docente en el proceso de enseñanza aprendizaje. UMBRAL. Revista de Educación, Cultura y Sociedad, 5(8), 170-172). Recuperado de http://sisbib. unmsm.edu.pe/BibVirtualdata/publicaciones/umbral/v05_n08/a21.pdf

Coll, C., Martín, E. y Onrubia, J. (2001). La evaluación del aprendizaje escolar: Dimensiones psicológicas, pedagógicas y sociales. En C. Coll, J. Palacios y A. Marchesi (Comps.). Desarrollo psicológico y educación 2. Psicología de la educación escolar (pp. 549-572). Madrid: Alianza.

De la Cruz Quintanilla, L. (2007). Diccionario de educación. Lima, Perú: s. e.

Espinal, C. A. (2003). La educación integral en las escuelas secundarias. México: Monografias.com. Recuperado de http://www.monografias.com/trabajos39/educacion-integral/educacionintegral2.shtml 
Gaitán, C., Campos, R., García, L., Granados, L., Jaramillo, L. y Panqueva, J. (2005). Prácticas educativas y procesos de formación en la educación superior. Bogotá: Pontificia Universidad Javeriana.

García, K. (2010). La evaluación como proceso de construcción de los aprendizajes. Medellín: [Editorial].

Hill, N. y Stone, W. C. (2003). La actitud mental positiva. Un camino hacia el éxito. Rosario Argentina. Edición digital Bilioteca de la Nueva Era.

Institución Educativa Técnica Comercial Caldas. (s. f.) Motivación hacia el aprendizaje. Recuperado de http://ietccaldasguamo.wordpress.com/directiva/clima-escolar/motivacion-hacia-elaprendizaje/

Ministerio de Educación Pública, República de Costa Rica (2007). Ley fundamental de educación. San José, Costa Rica, Autor. Recuperado de http://www.bcn.cl/carpeta temas_profundidad/temas_profundidad.2007-08-29.4296398389/legislacion-extranjera/ ley\%20fundamental\%20de\%20educacion_costa\%20rica\%20.pdf

Muñoz, B. (30 de enero de 2010). Desempeño docente Vs rendimiento escolar. [Mensaje en un blog] Tomado de http://evaluadoresacreditadores.blogspot.com/2010/01/desempenodocente-vs-rendimiento.html

Resolución № 0229 (03 diciembre, 1999). Colegio la eneñanza pre-escolar, básica primaria y bachillerato.de la Secretaría Distrital de Educación y Cultura de Cartagena. Recuperado de http://www.colegiolaensenanza.edu.i8.com/incentivos.htm

Reyes, L. A. (2006). Estándares de desempeño docente. Chile: Ediciones Universidad Católica Silva Henríquez. Santiago. Recuperado de http://www.oei.es/docentes/articulos/estandares_ desempeno_docente_reyes.pdf

Ruiz, J. C., Álvarez, N. y Pérez, E. (2008). La orientación socio-humanística, un aporte a la formación integral del estudiante. Tendencias Pedagógicas, 13, 175-191. Recuperado de www.tendenciaspedagogicas.com/Articulos/2008_13_09.pdf

\section{Cómo citar este artículo en APA:}

Quirós, E. y Jiménez, X. (2013). La actitud hacia la docencia, la formación integral en la educación secundaria y a su proyecto de vida: Una visión desde los estudiantes de colegios en áreas rurales. Revista Electrónica Educare, 17(2), 91-109. Recuperado de http://www.revistas.una.ac.cr/index.php/EDUCARE/issue/current

Nota: Para citar este artículo en otros sistemas puede consultar el hipervínculo "Como citar el artículo" en la barra derecha de nuestro sitio web:

http://www.revistas.una.ac.cr/index.php/EDUCARE/index 